\title{
Influence of droplet clustering in sprays on liquid deposition rate on spherical targets
}

\author{
Paul Andrade*, Georgios Charalampous, Yannis Hardalupas \\ Mechanical Engineering Department, Imperial College London, SW7 2AZ, UK \\ *Corresponding author: p.andrade14@imperial.ac.uk
}

\begin{abstract}
The origin of temporal fluctuations of liquid mass deposition rates, obtained from a spray of droplets impinging on a solid spherical target, was investigated by correlation with droplet clusters in the spray. The droplet clusters were quantified using a Voronoi analysis on instantaneous images of the droplets, to obtain the number of droplet clusters, the area of the clusters and the number of droplets in each cluster. It was found that the normalised area of the droplet clusters had a distribution with a peak around $10^{-1}$ and a right tail which followed a power law of exponent -1.8. As the number density of the droplets inside the clusters increased, the temporal fluctuations of the liquid mass deposition rates increased, as a greater variation of droplet sizes impinged the target. However, as the standard deviation of the distribution of the normalised droplet cluster areas was increased, the temporal fluctuations in the liquid mass deposition rates reduced, as variations to the droplet number density and droplet sizes inside the clusters were averaged out.
\end{abstract}

\section{Keywords}

Clustering, Spray Impingement, Liquid Deposition.

\section{Introduction}

Multi-phase flows occur in a wide range of natural and industrial applications, such as rain formation, atmospheric emission, spray drying and combustion. In many of these applications, the surrounding fluid might be turbulent and this may influence how the particles disperse in such systems. For instance, during spray drying, an industrial process which produces powder from a liquid slurry feed, the change in dispersion caused by the surrounding hot turbulent air may alter the rate of collisions which occur between droplets of the atomized slurry, as well the outcome of the collisions. This may then change the morphology of the produced powder [1], and so it is important therefore to gain physical insight into how these physical processes behave. Whilst a unified theory which describes completely the dispersion of particles in a turbulent flow is lacking, two non-dimensional numbers, the Stokes number and the Gravitational settling parameter, have been used to characterize the dispersion.

The Gravitational settling parameter, $\phi$, characterizes the influence of gravity to the dispersion of the particle and is defined in Equation (1) as the ratio of the terminal velocity of a particle, $\mathrm{V}_{\mathrm{T}}$, to a characteristic velocity scale of a turbulent flow, $V_{F}$, typically the Kolmogorov scale [2]. Particles with a high settling parameter may have little lateral dispersion as they fall. This is because these particles have a very high terminal velocity and therefore spend little time in local turbulent flow eddies, so their response to these eddies occurs over a reduced period of time [3]. Alternatively, particles with a very low settling parameter will spend more time in the local eddies and therefore may have greater ability to disperse.

$$
\phi=\mathrm{V}_{\mathrm{T}} / \mathrm{V}_{\mathrm{F}} .
$$

The Stokes number, St, characterizes the influence of the particle inertia to its ability to disperse in the fluid flow. It is typically defined as the ratio of two time scales, $\tau_{\mathrm{P}}$ and $\tau_{\mathrm{F}}$, as shown in Equation (2) [4]. ' $\tau_{\mathrm{P}}$ ' is the particle response time and is the time required for a particle to respond to the local turbulent velocity fluctuations. ' $\tau_{\mathrm{F}}$ ' is a characteristic time scale of the turbulent flow and, for small particles, the Kolmogorov time scale is typically selected as the characteristic time [5].

$$
\mathrm{St}=\tau_{\mathrm{P}} / \tau_{\mathrm{F}} .
$$

If particles have a very low Stokes number, i.e. St $<<1$, the particles offer little resistance to turbulent velocity fluctuations and only slightly deviate from the instantaneous streamlines of the flow. If St $>>1$, particles have a high inertia and therefore follow trajectories defined by their initial boundary conditions, ignoring the majority of the fluctuations imparted on them by the flow. If however, St $\sim 1$, the particles only partially respond to the local fluctuations. This leads to particles aggregating closer together, creating regions of the flow field where a large number of particles accumulate, forming 'clusters' and regions of the flow field where there are few particles, forming 'voids' [6]. Since clusters have a high particle density, they can increase the probability of collisions [7,8].

A wide range of Stokes numbers and Gravitational settling parameters may exist in multi-phase flows. One such example, is during operation of an industrial spray dryer. At the beginning of the process, an atomizer creates a polydispersed spray of droplets containing liquid, fine solids and air. As the droplets are dried by the surrounding hot turbulent air, the remaining particles become 'sticky', after their surface reaches a critical viscosity [9]. The turbulent 

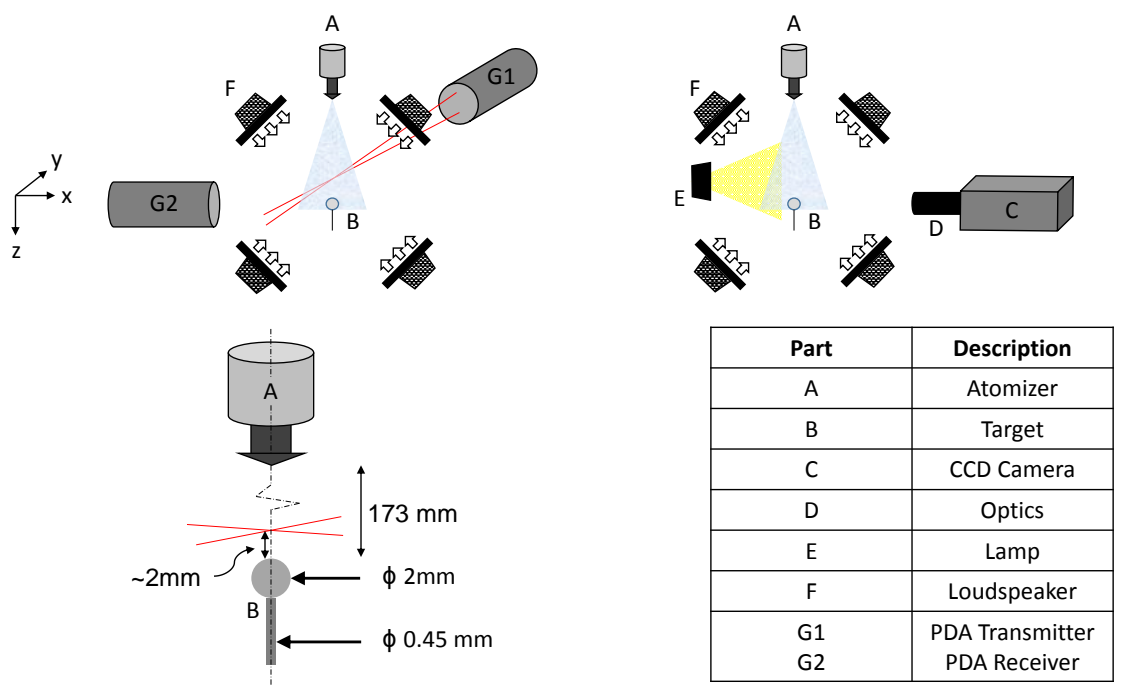

Figure 1. Details of the experimental set-up.

air flow promotes collisions between the different particles, with the outcome of the collisions dependent on the geometric and kinematic properties of particles, their trajectories and the surrounding air [10-13]. This can lead to formation of larger particles due to agglomeration, where a drying droplet or a sticky particle deposits on another sticky particle [9]. It can also produce much smaller particles due to the formation of 'secondary droplets' during droplet splashes on a sticky particle, causing a part of the droplet to re-atomise during impact [11]. The secondary droplets are then dried by the hot air to form 'fines'. These different physical processes lead to the formation of a wide range of droplet and particle sizes with variable composition inside the spray drier. Since the particle response time and terminal velocity, used to calculate St and $\phi$, depend on the droplet/particle size and composition, there must also be a wide range of Stokes numbers and settling parameters. It is therefore very difficult to assess how the particles disperse in the turbulent air flow inside the spray drier, even if the distribution of Stokes numbers and settling parameters is known. This makes the production of powders with desired characteristics quite inefficient.

To better understand the influence of the surrounding turbulent air on droplet/particle dispersion and particle-droplet collisions, imaging-based experiments of binary collisions involving a droplet impinging on a spherical solid target were carried out [13], inside a facility called the 'Box of Turbulence' [14]. This facility produces homogeneous isotropic turbulence without mean flow, allowing for the study of turbulent fluctuations on droplet dispersion. The surrounding turbulent air flow caused large lateral dispersion of the impinging droplets and changed the collision geometry, which influenced the outcome of the collisions. In more recent work [15], a spray, produced by an ultrasonic atomizer, impinged on the target, and imaging techniques were used to quantify the rate of accumulation of liquid on the target. The experiment was implemented inside the box of turbulence, which allowed the influence of the surrounding air turbulence on the rate of liquid depositing on the target to be investigated. As the intensity of the turbulent velocity fluctuations increased, the rate of liquid accumulation reduced. This is because fewer droplets from the spray impinged on the target, as they dispersed laterally due to the flow turbulence. Additionally, the collision outcomes may change due to the variation of the relevant parameters, as demonstrated by previous work on binary collisions.

It was noted that, for a fixed set of spray operating conditions, the temporal fluctuations of the accumulated liquid on the target increased with time. These temporal fluctuations propagated on the measurements of the liquid deposition rates. The aim of this paper is to investigate the origin of these fluctuations by correlating the estimated liquid deposition rates to characteristics of instantaneous clusters of droplets, which may occur in the spray. The paper is organized as follows. The next section describes the experimental set-up. This includes details of the characteristics of the droplets in the spray and the estimated liquid deposition rates, both of which were obtained previously but are relevant to the current paper. Also included in the methodology is a description of how recorded instantaneous images of droplets in the spray were processed using Voronoi analysis, which allowed droplet clusters to be identified. The following section presents the findings in terms of the droplet cluster characteristics, quantified from the Voronoi analysis, and correlates them to the deposition rate information on the solid target and discusses the droplet clustering implications on the time-dependent liquid deposition rates. The paper ends with a summary of the main findings.

\section{Methodology}

An experiment was set up inside the 'box of turbulence' facility, where a spray, produced by an ultrasonic atomizer, (Sono-Tek Ultrasonic $48 \mathrm{KHz}$ ) impinged on a spherical target (Figure 1). The target material was Stainless Steel 
316 , with a diameter of $2 \mathrm{~mm}$ and a maximum surface roughness of $0.125 \mu \mathrm{m} \mathrm{R}_{\mathrm{a}}$. The target was supported by a hypodermic needle (BD Microlance $326 \mathrm{G}$ ), of diameter $0.45 \mathrm{~mm}$. The box of turbulence uses eight loudspeakers mounted on a cubic frame to create synthetic jets. Each loudspeaker is directed towards the centre of the frame and homogeneous isotropic turbulence, without mean flow, is generated at the centre, as shown in Refs [14, 16]. The target was placed at the centre of the cubic frame, and the atomizer placed approximately $17.3 \mathrm{~cm}$ directly above the target, positioned just above the loudspeakers to avoid interference with the flow field. The flow field was characterized using the turbulent Reynolds number, $\mathrm{Re}_{\lambda}$, obtained from Particle Image Velocimetry (PIV) measurements of the flow field using a commercial system (LaVision). Three different Reynolds numbers were investigated, namely $\operatorname{Re}_{\lambda}=92,110$ and 136 .

The characteristics of the droplets in the spray were measured using Phase Doppler Anemometry (PDA), using a commercial system (Dantec Dynamics). The probe volume was placed just a few millimetres above the target. The droplet velocity and size were measured, from which the number density was also estimated. Depending on the intensity of the turbulent air and the atomization conditions, the droplet velocities were found to be around $1 \mathrm{~m} / \mathrm{s}$, the Sauter mean diameter of the droplets was approximately $40 \mu \mathrm{m}$ and the number density of the droplets were of the order of $10^{5}$ droplets per cubic centimetre. Since the atomizer imparted some momentum to the droplets, the instantaneous droplet velocity and turbulent flow velocity of the large eddies were used as characteristic velocities for the settling parameter. The settling parameter varied between approximately 13 and 55, whilst the Stokes numbers varied between approximately 0 to 1.4 .

A CCD camera then recorded images of the spray impinging on the target at a frame rate of $5 \mathrm{~Hz}$, with an exposure time of $1000 \mu \mathrm{s}$ and a magnification of approximately $6 \mu \mathrm{m} / \mathrm{px}$. The droplets deposited on the target, creating an initially thin liquid film around its surface. With further droplet impingements, the liquid film grew with time and drained underneath the target, before detaching due to gravity [15]. After detachment, the process was then repeated. The accumulation of liquid on the target was estimated using image processing (Matlab). First, Canny edge detection was used to detect the edges of the liquid film and the target. The edges were filled in, providing an estimate of the combined area of the target and the liquid film. The target area was deducted using a baseline image of a dry target. By assuming the liquid film developed symmetrically around the target, the liquid volume could be calculated. The mass of liquid, which accumulated around the target, was then plotted as a function of time. A fitting procedure was used to obtain the gradient of the line of best fit for the data, which corresponded to the liquid mass deposition rate. For a given experimental condition, since the process was repeated several times, several estimates of the liquid mass deposition rate were obtained.

In a separate experiment, for each experimental condition, 500 instantaneous droplet images were obtained in the spray, at a frame rate of $2.5 \mathrm{~Hz}$ and a magnification of $90 \mu \mathrm{m} / \mathrm{px}$. For the current work, a Voronoi analysis was applied to the droplet images of the spray (Matlab), as described by Ref [17], and quantified the number of droplet clusters present at each image, the number of droplets in each cluster and the area of each cluster. First, droplets below a given intensity threshold were removed from the image. This is because the images were obtained through illumination by a laser sheet and droplets out of the plane of the laser sheet, corresponding to low light scattering intensity droplets, could contribute adversely to the analysis. Next, for the remaining droplets, the Voronoi cells were computed. The boundary of each Voronoi cell defined a region of points on the image, which were closest to a given droplet. In other words, if a location on the image was chosen at random, the nearest droplet to that location is immediately known by virtue of the Voronoi cell and therefore, by definition, each Voronoi cell must contain only one droplet. If the droplets on the image were located close together, the Voronoi cells around these droplets were small. If droplets on the image were spaced far apart, their Voronoi cells would be larger. By using the area of the Voronoi cell as a measure of its size, clusters of droplets were identified based on connected Voronoi cells whose area fell below a given threshold. The threshold used is based on the intersection of the distribution of Voronoi cells in the image with a Poisson distribution of Voronoi cells of the same particles [17]. The latter distribution represents a random spatial distribution of droplets in the image domain and is obtained using the $2 \mathrm{D}$ analytical result by Ref [18].

\section{Results \& Discussion}

The results are presented in two sections. In the first section, the characteristics of the droplet clusters in the spray are quantified, when the surrounding gas is quiescent or turbulent. This then provides statistics which can be used to correlate to the liquid deposition rates in the second section.

\section{Quantification of droplet cluster characteristics in the spray}

The probability density function (PDF) for the detected area of the droplet clusters in the spray from all images for a given experimental condition was computed using a histogram consisting of 1000 bins (Figure 2). The area of each cluster, denoted $A_{C}$, was normalized with the average cluster area, $\overline{A_{C}}$. The distribution of the area of the droplet clusters displays a peak at around $10^{-1}$ for all Reynolds number. The results for liquid atomization by the ultrasonic atomiser driven by powers of $2.7 \mathrm{~W}$ and $3.2 \mathrm{~W}$ at the same liquid flow rate are qualitatively similar. Some authors have observed power law distributions with exponents of $-5 / 3$ to -2 for the probability of the droplet cluster areas [17, 19, $20]$, or of the qualitatively equivalent voids [17, 20-22]. However, a power-law distribution has only a straight line on log-log axes, but some of the aforementioned authors also observed peaks in their droplet cluster/void distributions $[17,19,20]$. A power law, with an exponent of -1.8 , is also shown in Figure 2 for comparison and appears to match 


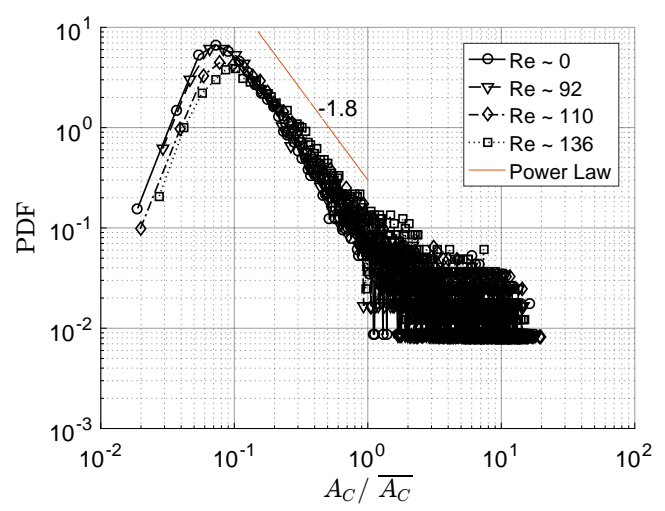

Figure 2. PDF of area of droplet clusters for operation of the ultrasonic atomiser with power and liquid flow rate of $2.2 \mathrm{~W}$ and 50 $\mathrm{cm}^{3} /$ min respectively.

the data only on the right tail of the distribution. A power law probability distribution of the droplet cluster area would suggest that droplet clusters are self-similar, implying that the clustering effect is due to a range of turbulent flow eddy sizes and not wholly dependant on the smallest eddies quantified by the corresponding Kolmogorov scales only [21].

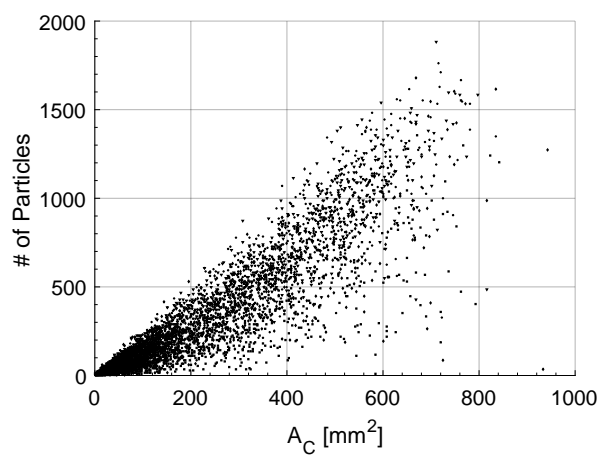

(a) Linear axes showing magnitude of variation

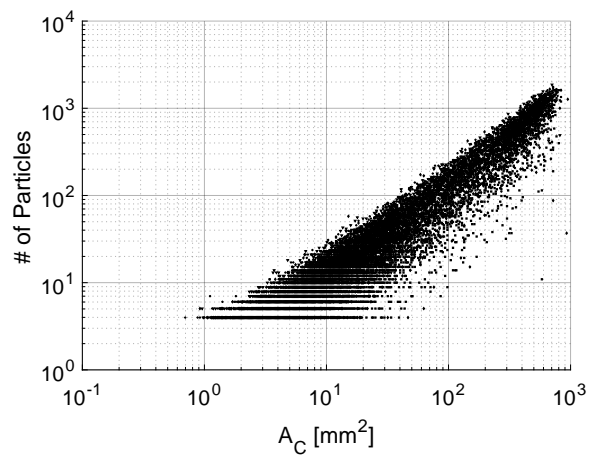

(b) Log-Log axes showing relative variation

Figure 3. Number of droplets detected in each cluster, for all Reynolds number, for the spray from the ultrasonic atomiser operating with power of $2.2 \mathrm{~W}$ and a liquid flow rate of $50 \mathrm{~cm}^{3} / \mathrm{min}$.

Next, the number of droplets inside each cluster was evaluated. Generally, the number of detected particles per cluster increased, as the area of the identified cluster increased (Figure 3). Whilst, for clusters with a large area, there was a greater magnitude of variation in the number of detected droplets (Figure 3a), the change in the number of detected droplets was actually more significant for clusters with a smaller area (Figure $3 \mathrm{~b}$ ).

Figure 3 may be explained physically by the response and dispersion of droplets to the local flow fluctuations. The number of droplets inside a cluster will depend on first, how many droplets existed in the neighbourhood of a cluster, at a short time interval, $\delta \tau$, before the cluster was formed (Figure 4). The response of the droplets to the local flow velocity fluctuations then determines which droplets will aggregate to form a cluster and which droplets do not. Since there is a wide distribution of Stokes numbers and settling parameters in the spray, there is a wide variation of response times to flow velocity fluctuations, influencing how many of those droplets, in a neighbourhood of the spray, aggregate to form the cluster.

If a cluster has a small area, it only covers a small region of the flow field. Prior to the formation of the small cluster, any droplets in its local neighbourhood may have only been exposed to a small range of flow velocity fluctuations (Figure 4a and Figure 4b). In Figure 4a, two droplets do not aggregate to form the cluster whereas in Figure 4b, four droplets do not aggregate to form the cluster. Whilst the change in the number of droplets aggregating is small (Figure 3a), the relative variation, i.e. the change in the number of droplets relative to the size of the cluster, is significant (Figure 3b). Alternatively, if a cluster has a large area, it covers a large region of the flow field. The droplets inside the large cluster may, therefore, have been exposed to a much wider range of flow velocity fluctuations before they aggregated into the cluster (Figure 4c). This in turn means that there may have been significant variations to how the different droplet sizes responded to different intensity of velocity fluctuations, leading to a large magnitude of variation in the number of droplets that formed the cluster, as shown in Figure 3a. However, since the cluster is so large, even if several droplets do not aggregate to form the cluster, its local number density is still high and, as a result, its relative variation is low (Figure $3 b$ ). 


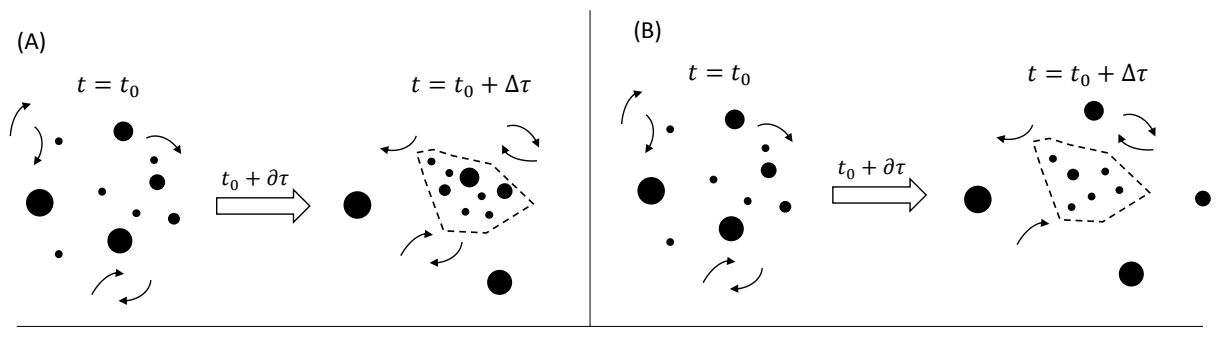

(C)
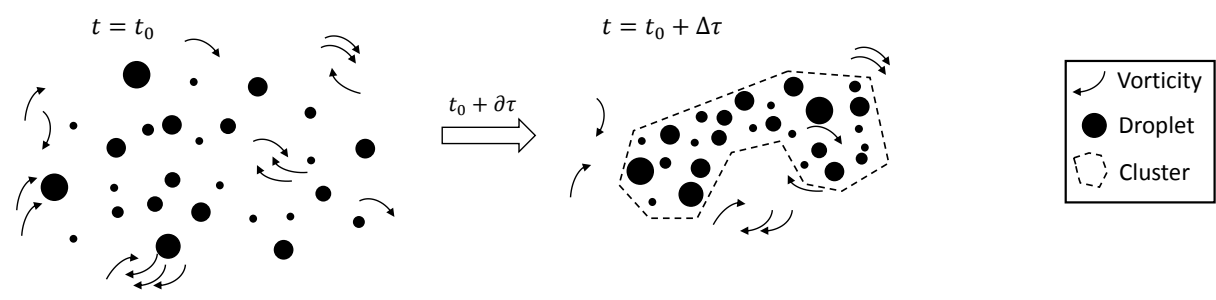

Figure 4. Schematic of cluster formation in polydispersed spray where ' $\mathrm{t}$ ' is the time, $t_{0}$ is the time as the cluster begins to form and $\delta \tau$ is a short interval for the cluster to form. (A) A group of droplets forms a small cluster. Two droplets do not aggregate into the cluster. (B) A group of droplets forms a small cluster, but several droplets do not aggregate into the cluster. (C) A group of droplets form a large cluster. The larger cluster may be formed from a larger range of local flow velocity fluctuation intensities.

Table 1. Coefficient of Determination values, $R^{2}$, for linear regression fit of number of droplets against area of cluster

\begin{tabular}{c|cccc}
\hline & $\operatorname{Re} \sim 0$ & $\operatorname{Re} \sim 92$ & $\operatorname{Re} \sim 110$ & $\operatorname{Re} \sim 136$ \\
\hline $2.2 \mathrm{~W}$ & 0.94 & 0.95 & 0.89 & 0.81 \\
$2.7 \mathrm{~W}$ & 0.90 & 0.90 & 0.88 & 0.79 \\
$3.2 \mathrm{~W}$ & 0.85 & 0.87 & 0.83 & 0.69
\end{tabular}

A linear regression fit was attempted to obtain the gradient of the number of droplets against the area of the cluster from Figure 3. The Coefficient of Determination values for the regression lines, $R^{2}$, are shown in Table 1 and the gradients of the regression line, denoted $\mathrm{D}_{\mathrm{ND}}$, are shown in Table 2. As the Reynolds number of the surrounding gas is increased, $R^{2}$ is reduced, suggesting the linear fit becomes less appropriate. This is true, regardless of the conditions used to generate the spray. As the Reynolds number is increased, the droplets have a greater lateral dispersion. If the atomization conditions are kept constant, the greater dispersion may lead to a greater variation of distances between droplets. This results in droplet clusters with a wider spread in the number of droplets they contain, which corresponds to a lower $\mathrm{R}^{2}$ value. The greater lateral dispersion means fewer droplets may also be located in the vicinity of the target and, therefore, there may be, for a cluster with a given size, fewer droplets inside the cluster, as reflected by $D_{N D}$ reducing with increasing Reynolds number in Table 2.

The frequency at which various sized clusters impinged on the target was also investigated. Since different cluster areas contain different number of droplets (Figure 3), the local droplet number density in each cluster may differ. The number density for each cluster was estimated by first, calculating the total number of droplets inside the cluster, $\sum N_{P}$ and then, dividing this by the cluster area, $A_{C}$. The clusters in each image of a given experimental condition were then 'binned' according to their area. The number density in these binned clusters was calculated and their frequency of occurrence was plotted (Figure 5). The liquid flow rate and the power supplied to the atomizer were kept fixed $\left(50 \mathrm{~cm}^{3} / \mathrm{min}\right.$ and $2.2 \mathrm{~W}$ respectively). It appears that very small clusters are more frequent than very large clusters (c.f. Figure $5 \mathrm{a}$ and Figure 5d). For a given Reynolds number, the droplet number density of the clusters is consistent regardless of the size of the cluster though as before (Figure $3 b$ ), there is greater relative variation in smaller cluster areas.

Table 2. Gradients, $D_{\mathrm{ND}}$, from the linear regression fit of number of droplets against area of cluster

\begin{tabular}{c|cccc}
\hline & $\operatorname{Re} \sim 0$ & $\mathrm{Re} \sim 92$ & $\mathrm{Re} \sim 110$ & $\mathrm{Re} \sim 136$ \\
\hline $2.2 \mathrm{~W}$ & 1.81 & 1.79 & 1.43 & 1.05 \\
$2.7 \mathrm{~W}$ & 1.35 & 1.34 & 1.20 & 0.89 \\
$3.2 \mathrm{~W}$ & 1.21 & 1.15 & 1.01 & 0.72
\end{tabular}




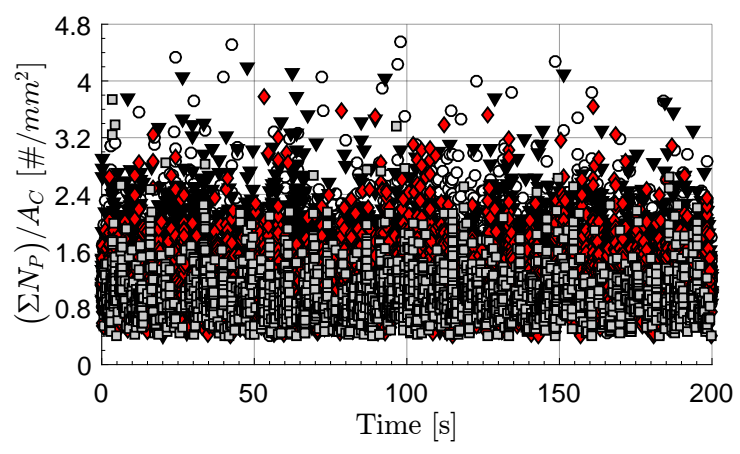

$\circ \operatorname{Re} \sim 0 \nabla \operatorname{Re} \sim 92 \diamond \operatorname{Re} \sim 110 \quad \square \operatorname{Re} \sim 136$

(a) $0 \mathrm{~mm}^{2} \leqslant A_{C}<10 \mathrm{~mm}^{2}$

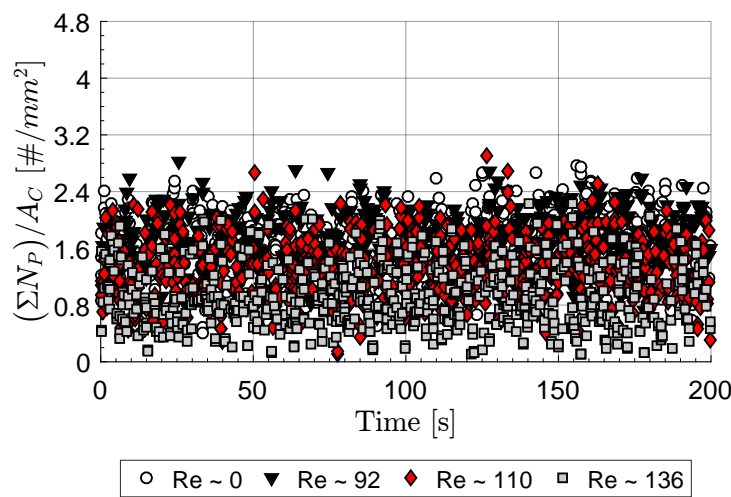

(c) $100 \mathrm{~mm}^{2} \leqslant \mathrm{~A}_{\mathrm{C}}<500 \mathrm{~mm}^{2}$

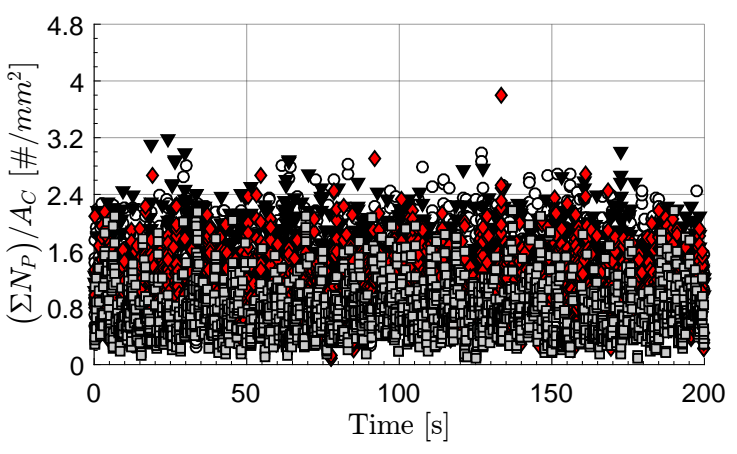

$\circ \operatorname{Re} \sim 0 \quad \boldsymbol{R e} \sim 92 \diamond \operatorname{Re} \sim 110 \quad$ a $\operatorname{Re} \sim 136$

(b) $10 \mathrm{~mm}^{2} \leqslant \mathrm{~A}_{\mathrm{C}}<100 \mathrm{~mm}^{2}$

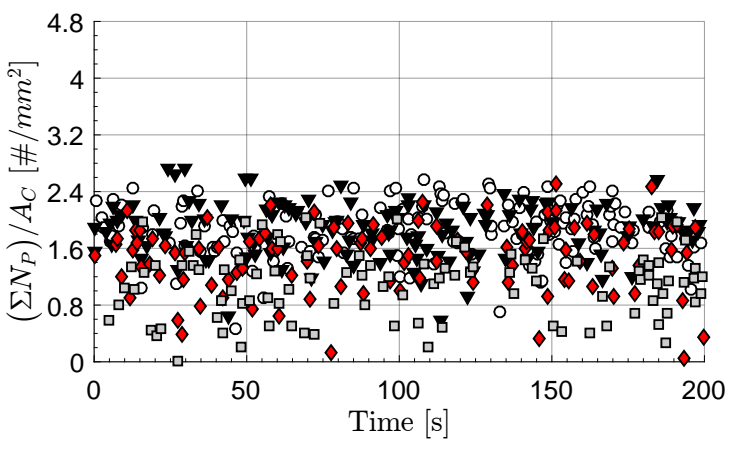

$\circ \operatorname{Re} \sim 0 \quad \boldsymbol{R e} \sim 92 \diamond \operatorname{Re} \sim 110 \quad \square \quad \operatorname{Re} \sim 136$

Figure 5. The relationship between the estimated number of droplets contained in each cluster, $\left(\sum N_{P}\right) / A_{C}$, and their probability of occurrence during $\sim 200$ seconds (500 images) of measurements.

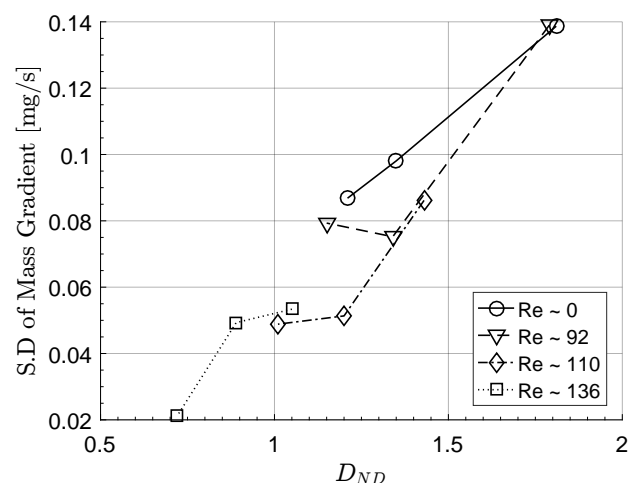

(a) Variation of liquid mass temporal gradients with gradients from Table 2.

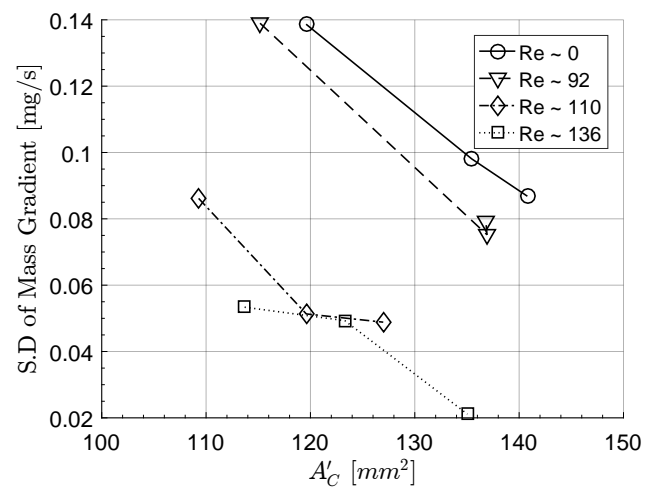

(b) Variation of liquid mass temporal gradients with standard deviation of cluster area, $A_{C}^{\prime}$.

Figure 6. Variation of standard deviation (S.D) of liquid mass temporal gradients with statistics from the clusters at different atomizer operating powers and at a fixed liquid flow rate of $50 \mathrm{~cm}^{3} / \mathrm{min}$ supplied to the atomizer. 


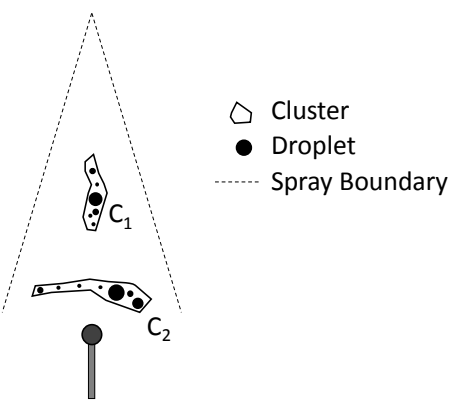

Figure 7. Droplet clusters may have different orientations relative to the target. This may influence the liquid deposition rate. Cluster $\mathrm{C}_{1}$ may deposit more droplets than $\mathrm{C}_{2}$, provided its properties do not change prior to impinging on the target.

\title{
Relationship between droplet clusters and liquid deposition rate
}

The influence of the droplet clusters to the estimated liquid deposition rates was then assessed and presented in Figure 6. Figure $6 a$ shows the standard deviation of the liquid mass temporal gradients increases as $D_{\mathrm{ND}}$ increases. $D_{N D}$ represents the average droplet number density of the clusters, for a given experimental condition. As the number density increases, there may be greater variation to the size of droplets inside the cluster. This may result in greater variation to the amount of liquid, which deposits on the target, corresponding to an increase to the spread of the liquid mass temporal gradients. Figure $6 \mathrm{~b}$ shows the standard deviation of the liquid mass temporal gradients decreases as the standard deviation of the area of the clusters, denoted by $A_{C}^{\prime}$, is increased. ' $A_{C}^{\prime}$ ' represents the spread of the distribution of the area of the clusters. If the spread is increased, a greater range of droplet cluster areas impinges on the target. This means any variations to the droplet number density (and the size of the droplets) inside the cluster are averaged out, leading to less variation of the liquid mass temporal gradients.

The shape and orientation of the cluster, relative to the target, as well as the size of the droplets inside the cluster must also be considered. If all the droplets were of similar size inside a cluster and all impinged on the target, Figure 5 may suggest that the intermittent clusters lead to temporal variations in how the liquid accumulates on the target. However, as well as there actually being a wide rage of droplet sizes in the spray, the shape and orientation of each cluster, relative to the target, can also vary substantially, and may be gravity dependant [23]. This may result in only part of a droplet cluster impinging on the target as shown in Figure 7. Provided that the clusters in the figure do not vary before they impinge on the target, most of the droplets contained in cluster $\mathrm{C}_{1}$ will deposit on the target, whereas only part of the droplets contained in $\mathrm{C}_{2}$ will impinge on the target.

\section{Conclusions}

The temporal fluctuations of the rate of liquid mass accumulation on a spherical target during spray impingement were studied by assessing the influence of characteristics of instantaneous droplet clusters formed in the spray. The main findings are summarized below:

- For a given Reynolds number, both large and small clusters appear to have similar local droplet number densities. Large clusters are much less frequent than small clusters. This may cause some of the variations to the rate of liquid deposition on the target.

- The greater the number density of droplets inside a cluster, the greater the fluctuations of the rate of liquid accumulation on a target.

\section{Acknowledgements}

We would like to acknowledge financial support from Procter and Gamble through an EPSRC industrial case studentship and EPSRC grant EP/K019732/1.

\author{
Nomenclature \\ $A_{C} \quad$ Area of Cluster $\left[\mathrm{mm}^{2}\right]$ \\ $A_{P} \quad$ Area of Particle $\left[\mathrm{mm}^{2}\right]$ \\ $\overline{A_{C}} \quad$ Mean Cluster Area $\left[\mathrm{mm}^{2}\right]$ \\ $\widetilde{A_{C}} \quad$ Standard Deviation of Cluster Area $\left[\mathrm{mm}^{2}\right]$ \\ Re Turbulent Reynolds Number \\ St Stokes Number
}

\section{References}

[1] D. F. Fletcher et al. "What is important in the simulation of spray dryer performance and how do current CFD models perform?" In: Applied Mathematical Modelling 30.11 (2006), pp. 1281-1292. ISSN: 0307-904x. DOI: DOI10.1016/j.apm.2006.03.006. 
[2] A. Aliseda et al. "Effect of preferential concentration on the settling velocity of heavy particles in homogeneous isotropic turbulence". In: Journal of Fluid Mechanics 468 (2002), pp. 77-105. ISSN: 0022-1120. DOI: Doi10. 1017/S0022112002001593.

[3] M. R. Wells and D. E. Stock. "The effects of crossing trajectories on the dispersion of particles in a turbulent flow". In: Journal of Fluid Mechanics 136 (1983), pp. 31-62. ISSN: 1469-7645. DOI: doi : 10 . 1017 / S0022112083002049.

[4] C. T. Crowe, R. A. Gore, and T. R. Troutt. "Particle Dispersion by Coherent Structures in Free Shear Flows". In: Particulate Science and Technology 3.3-4 (1985), pp. 149-158. ISSN: 0272-6351. DOI: 10. 1080/02726358508906434.

[5] A. M. Wood, W. Hwang, and J. K. Eaton. "Preferential concentration of particles in homogeneous and isotropic turbulence". In: International Journal of Multiphase Flow 31.10-11 (2005), pp. 1220-1230. ISSN: 0301-9322. DOI: DOI10.1016/j.ijmultiphaseflow .2005.07.001.

[6] R. Monchaux, M. Bourgoin, and A. Cartellier. "Analyzing preferential concentration and clustering of inertial particles in turbulence”. In: International Journal of Multiphase Flow 40 (2012), pp. 1-18. ISSN: 0301-9322. DOI: DOI10.1016/j.ijmultiphaseflow .2011.12.001.

[7] S. Sundaram and L. R. Collins. "Collision statistics in an isotropic particle-laden turbulent suspension .1. Direct numerical simulations”. In: Journal of Fluid Mechanics 335 (1997), pp. 75-109. ISSN: 0022-1120. DOI: Doi10. 1017/S0022112096004454. URL: \%3CGo\%20to\%20ISI\%3E : //WOS : A1997WW06300004.

[8] W. C. Reade and L. R. Collins. "Effect of preferential concentration on turbulent collision rates". In: Physics of Fluids 12.10 (2000), pp. 2530-2540. ISSN: 1070-6631. DOI: Doi10.1063/1.1288515. URL: \%3CGo\%20to\% 20ISI\%3E : //WOS : 000089171000016.

[9] R. E. M. Verdurmen et al. "Agglomeration in Spray Drying Installations (The EDECAD Project): Stickiness Measurements and Simulation Results". In: Drying Technology 24.6 (2006), pp. 721-726. ISSN: 0737-3937. DOI: $10.1080 / 07373930600684973$.

[10] J. Qian and C. K. Law. "Regimes of coalescence and separation in droplet collision". In: Journal of Fluid Mechanics 331 (1997), pp. 59-80. ISSN: 1469-7645. DOI: doi : 10.1017/S0022112096003722.

[11] Y. Hardalupas, A. M. K. P. Taylor, and J. H. Wilkins. "Experimental investigation of sub-millimetre droplet impingement on to spherical surfaces". In: International Journal of Heat and Fluid Flow 20.5 (1999), pp. 477485. ISSN: 0142-727X. DOI: http://dx.doi .org/10.1016/S0142-727X (99) 00045-4.

[12] A. L. Yarin. "Drop impact dynamics: Splashing, spreading, receding, bouncing..." In: Annual Review of Fluid Mechanics 38 (2006), pp. 159-192. ISSN: 0066-4189. DOI: DOI10.1146/annurev .fluid.38.050304.092144.

[13] G. Charalampous and Y. Hardalupas. Droplet-Particle Collisions and Breakup in Homogenous and Isotropic Turbulence. 27th European Conference on Liquid Atomization and Spray Systems. Sept. 2016.

[14] G. Charalampous and Y. Hardalupas. Preferential concentration of water droplets in a volume of homogeneous and isotropic turbulence. 49th AIAA Aerospace Sciences Meeting including the New Horizons Forum and Aerospace Exposition. Jan. 2011.

[15] P. Andrade, G. Charalampous, and Y. Hardalupas. Spray impingement on spherical targets in homogenous isotropic turbulence. 27th European Conference on Liquid Atomization and Spray Systems. Sept. 2016.

[16] H. Lian, G. Charalampous, and Y. Hardalupas. "Preferential concentration of poly-dispersed droplets in stationary isotropic turbulence". In: Experiments in Fluids 54.5 (2013). ISSN: 0723-4864. DOI: Artn1525Doi10. 1007/S00348-013-1525-3.

[17] R. Monchaux, M. Bourgoin, and A. Cartellier. "Preferential concentration of heavy particles: A Voronoi analysis". In: Physics of Fluids 22.10 (2010). ISSN: 1070-6631. DOI: Doi10.1063/1.3489987.

[18] F. Jarai-Szabo and Z. Neda. "On the size-distribution of Poisson Voronoi cells". In: eprint arXiv:cond-mat/0406116 (June 2004). eprint: cond-mat/0406116.

[19] M. Obligado et al. "Preferential concentration of heavy particles in turbulence". In: Journal of Turbulence 15.5 (2014), pp. 293-310. ISSN: 1468-5248. DOI: Doi10.1080/14685248.2014.897710.

[20] Sholpan Sumbekova et al. "Preferential concentration of inertial sub-Kolmogorov particles: The roles of mass loading of particles, Stokes numbers, and Reynolds numbers". In: Physical Review Fluids 2.2 (2017), p. 024302. DOI: 10.1103/PhysRevFluids.2.024302.

[21] S. Goto and J. C. Vassilicos. "Self-similar clustering of inertial particles and zero-acceleration points in fully developed two-dimensional turbulence". In: Physics of Fluids 18.11 (2006). ISSN: 1070-6631. DOI: Artn115103Doi10. 1063/1.2364263.

[22] G. Boffetta, F. De Lillo, and A. Gamba. "Large scale inhomogeneity of inertial particles in turbulent flows". In: Physics of Fluids 16.4 (2004), pp. L20-L23. ISSN: 1070-6631. DOI: Doi10.1063/1.1667807.

[23] A. Dejoan and R. Monchaux. "Preferential concentration and settling of heavy particles in homogeneous turbulence". In: Physics of Fluids 25.1 (2013). ISSN: 1070-6631. DOI: Doi10.1063/1.4774339. 